\title{
Hegemonía y contrahegemonía en el fenómeno youtuber
}

\author{
ISRAEL MÁRQUEZ Y ELISENDA ARDĖVOL
}

En un corto periodo, los youtubers -jóvenes usuarios de internet que han alcanzado cierta fama por los videos que publican en YouTube - se han convertido en nuevos modelos a seguir para niños y jóvenes, el tipo de público que habitualmente ve, sigue y comenta sus videos. Durante los últimos años, el fenómeno de los youtubers -y YouTube en conjunto- ha sufrido un proceso de "colonización" o "apropiación" capitalista, impulsado por grandes corporaciones mediáticas, que parecen reproducir la situación de control y hegemonía cultural no muy diferente de la era de la comunicación masiva. Sin embargo, es propio de toda hegemonía ser desafiada y resistida. Los youtubers estudiados se rebelan contra este poder hegemónico o critican de manera abierta estas nuevas formas culturales en las que se ven inmersos con recursos contrahegemónicos, como el humor y la ironía.

PALABRAS CLAVE: YouTube, youtubers, audiencia, hegemonía, contrahegemonía, juventud, censura

\section{Hegemony and Counterhegemony in the Youtuber Phenomenon}

ISRAEL MÁRQUEZ

Investigador posdoctoral "Juan de la Cierva", Ministerio de Economía, Industria y Competividad/Universitat Oberta de Catalunya, Barcelona, España isravmarquez@gmail.com

ELISENDA ARDĖVOL Universitat Oberta de Catalunya, Barcelona, España eardevol@uoc.edu
In a short period, youtubers-young Internet users who have reached a certain level of fame by publishing videos on YouTube-have become new models for children and young people, the public who watch, follow and comment their videos. Over the last few years, the youtuber phenomenon-and YouTube as a whole-has been reached by a process of capitalist "colonization" or "appropriation" driven by large media corporations, which seems to be reproducing a situation of control and cultural hegemony not very different from the mass communication era. However, hegemony is continually challenged and resisted, and the youtubers we studied rebel against this hegemonic power or openly criticize the new cultural forms in which they are immersed using counterhegemonic resources such as humor and irony.

KEYWORDS: YouTube, youtubers, audience, hegemony, counterhegemony, youth, censorship 


\section{Introducción*}

YouTube es uno de los sitios digitales más populares en la actualidad. Desde su lanzamiento en 2005, se ha convertido en un espacio privilegiado para el almacenamiento, difusión y consumo de todo tipo de contenidos, desde videos de aficionados hasta contenido audiovisual más profesional. La plataforma es, además, el segundo buscador más utilizado del mundo, sólo superado por Google, empresa a la que pertenece. YouTube, al igual que otras redes sociales como Facebook o Twitter, se ha convertido en un espacio digital omnipresente en la vida cotidiana de muchas personas. Millones de usuarios alrededor del mundo han incorporado YouTube y prácticas como compartir, ver, subir y comentar videos a sus hábitos y rutinas diarias, en especial las generaciones más jóvenes. Algunos de ellos incluso han sabido sacar provecho de esta red social y utilizarla como un medio para hacerse famoso y ganar dinero. Éste es el caso de los denominados youtubers o youtuberos, un término que se ha extendido últimamente en internet y los medios tradicionales y que ha pasado a formar parte del vocabulario y el imaginario popular. Su éxito entre el público joven ha sorprendido a los medios tradicionales y su creciente fama es un elemento controvertido en la configuración de nuevos públicos emergentes en muchos países.

¿Qué es en realidad un youtuber? En principio, un youtuber puede ser toda aquella persona que sube videos a YouTube, independientemente de su contenido, calidad y alcance. Sin embargo, la imagen que más se ha generalizado de un youtuber es la de un usuario de internet, por lo general joven, que sube videos a la plataforma de manera regular, cuenta con un número elevado de suscriptores y además consigue ganar dinero por publicar videos. Según esta definición —la más difundida por los medios de comunicación-, un youtuber sería en específico la persona que ha conseguido hacer de YouTube un medio para ganarse la vida, y en

Este artículo forma parte de una investigación en curso sobre jóvenes y cultura digital, desarrollada en el marco del proyecto Transmedia Literacy, Exploiting Transmedia Skills and Informal Learning Strategies to Improve Formal Education, auspiciado por el programa Horizon 2020 de la Unión Europea (H2O20-ICT-2014-1 645238). 
algunos casos, su verdadera profesión. Hay muchos tipos de youtubers, según su área de expertise, que pueden ir desde tecnología, hasta cocina, bricolaje o moda, pero los más populares entre los jóvenes, que acaparan audiencias por millones, son los denominados gameplayers - jugadores que comentan sus partidas de videojuegos y hablan de videojuegos en general- y los que utilizan el humor y la ironía para contar historias, por lo regular sobre temas de la vida cotidiana o el propio universo youtuber.

El término youtuber a veces se confunde con otro anglicismo que también se ha popularizado en tiempos recientes, utilizado en especial en el ámbito del marketing digital y las redes sociales. Nos referimos al término influencer, que se refiere a una persona influyente que por medio de opiniones y comentarios expresados en sus redes sociales consigue influir y movilizar a un gran número de seguidores. Un youtuber se convierte en influencer cuando su capacidad de movilizar a la audiencia va más allá de YouTube y se extiende hacia otras redes sociales y sitios web, incluso más allá del ámbito digital, en ferias de videojuegos, espectáculos de teatro, presentaciones de libros, etc. Algunos youtubers se han convertido en verdaderos influencers, como demuestran casos paradigmáticos, como el sueco PewDiePie, durante mucho tiempo el usuario con el mayor número de suscriptores de YouTube: más de 50 millones, el chileno HolaSoyGerman, con más de 30 millones de suscriptores, o el español El Rubius, con más de 20 millones. En estos casos, estamos ante nuevos fenómenos de masas gestados a partir de internet y la web 2.0, en los que la centralidad de YouTube como plataforma de expresión, creación y mantenimiento de comunidad ha sido determinante.

En el caso específico de España, existen muchos otros youtubers, hombres y mujeres, además de El Rubius, que han conseguido alcanzar cierta fama y popularidad gracias a sus canales de YouTube. Algunos ejemplos son Vegeta777, Willyrex,
AuronPlay, Wismichu, Jordi Wild, Dulceida, YellowMellowMG, Sarinha, alexelcapo, DaniRep, DalasReview, Celopan, Ter, etc. Se trata de canales que acumulan un gran número de visitas y seguidores de varios países iberoamericanos, cuyo contenido va del humor negro a los videojuegos, pasando por comentarios y opiniones sobre moda, actualidad, o consejos sobre hechos y situaciones de la vida cotidiana. Lo que tienen en común estos youtubers no es el contenido de sus videos, sino haber sabido conectar una manera original, auténtica y creativa con un amplio número de usuarios para crear su propia audiencia.

En este artículo estudiaremos el caso de estos youtubers españoles que se dirigen a un público joven, entre 12 y 25 años de edad, en el marco del proyecto europeo Transliteracy (2015-2018), centrado en el estudio de las habilidades transmedia y el aprendizaje informal entre adolescentes. Los datos se tomaron entre octubre de 2016 y mayo de 2017 , en talleres y entrevistas con adolescentes de 12 a 18 años de edad y en trabajo de campo basado en la observación de la actividad en la red. En este estudio, realizado en ocho países - Uruguay, Colombia, España, Portugal, Italia, Reino Unido, Finlandia y Australia-, se detectó la importancia de los youtubers en el consumo mediático global de la juventud y sus roles como forma de entretenimiento y aprendizaje. Presentamos una reflexión sobre este fenómeno a partir de nuestra exploración personal para el caso de algunos youtubers españoles que los jóvenes identifican como sus preferidos. En concreto, nos interesa destacar, por una parte, cómo se construye la narración o "estilo youtuber" a partir de lo que Lafkioui (2008) denomina una "oralidad multimodal", que se enlaza con otras fuentes más canónicas de la retórica (Leith, 2012) y con la vivacidad de la narración oral (Benjamin, 1991). Por otra parte, nos interesa conocer su economía política, es decir, cómo el fenómeno youtuber se inscribe en un contexto de lucha por la hegemonía cultural, cómo se posicionan los 


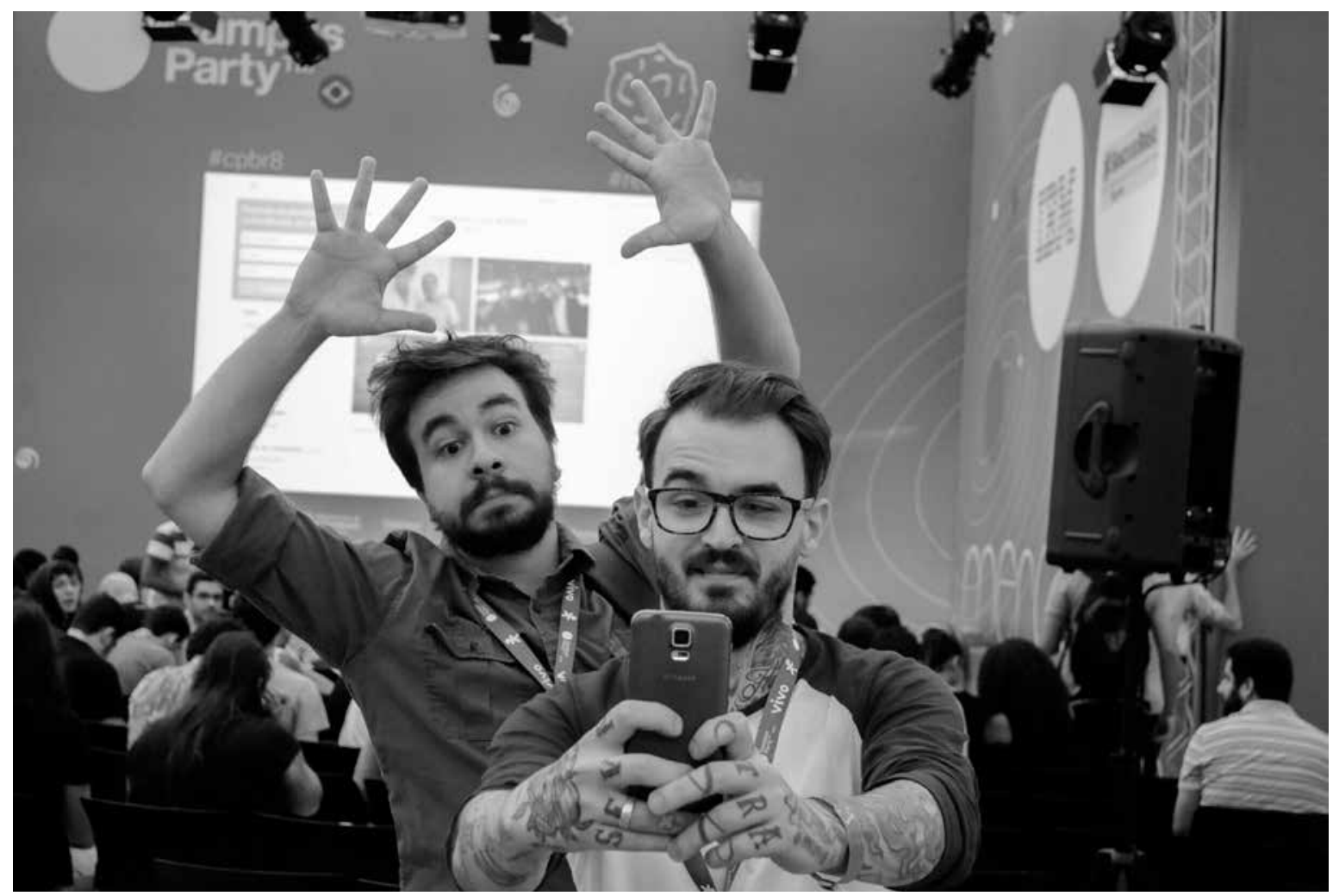

Daniel Nascimento Youtubers en Campus Party Brasil, 2015.

youtubers estudiados en relación con el mercado y cuáles son las nuevas formas de poder hegemónico y contrahegemonías que se esbozan en este contexto (Martín-Barbero, 1987; Williams, 2000).

\section{Construir audiencias: el estilo youtuber}

Argumentamos que existe una especie de "estilo youtuber" particular, si podemos llamarlo así, que ha permitido a estos jóvenes creadores de contenido audiovisual construir su audiencia y mantener la fidelidad de sus seguidores en sus canales de YouTube y más allá de ellos. Esta audiencia, conformada en su mayoría por gente joven, ve a los youtubers como nuevos referentes, ídolos e incluso líderes de opinión, que hablan su mismo lenguaje y tratan temas que les interesan y los "tocan" de manera íntima y personal, que rara vez encuentran en medios de comunicación tradicionales, como la prensa, la radio o la televisión. De ahí que en la actualidad, para esta joven audiencia youtubera, este espacio se haya convertido en una especie de nueva televisión que les habla e interpela de una manera que entienden como auténtica, natural, espontánea y directa.

El modo en que los youtubers se comunican e interactúan con su audiencia puede entenderse como un estilo narrativo propio de la cultura digital, en el que se mezclan diversos aspectos significativos de la oralidad, que se encarnan en un entorno digital y audiovisual específico que admite otras formas expresivas — gráficas, audio, texto, performances-, de ahí que pueda caracterizarse como una forma de “oralidad multimodal” (Lafkioui, 2008). 
En primer lugar, este estilo youtuber tiende a favorecer modos orales de narración. Los youtubers son, ante todo, personas que cuentan historias y lo hacen de manera que consiguen cautivar y atrapar a su audiencia. Con este propósito, los youtubers recurren a un lenguaje coloquial, en ocasiones vulgar, soez y ordinario, que consigue conectar de manera eficaz con el público joven que habitualmente ve, sigue y comenta sus videos. Ya sean consejos sobre la vida cotidiana o comentarios sobre un determinado videojuego, los youtubers construyen en cada video un relato oral directo que conecta emocionalmente con su audiencia. Más que transmitir el relato, el youtuber implica a su audiencia en él, para lo que recurre a fórmulas inclusivas como "hoy quería contaros", "os voy a decir una cosa", "me interesa lo que opináis", etc. En ocasiones, los youtubers incluso piden a sus seguidores que sugieran el tema que tratará en su próxima entrega: "he hecho este video porque muchos me lo habéis pedido", etc. Así, el youtuber establece una relación de cercanía y confianza con su audiencia, y va construyendo una especie de relación cara a cara, mediada digitalmente, en la que la oralidad juega un papel fundamental.

Por otro lado, la propia interfaz de YouTube favorece este tipo de relación de proximidad al permitir a los usuarios opinar, compartir y comentar los videos que se suben y almacenan en la plataforma. La audiencia muestra apoyo, acuerdo o desacuerdo con los videos, sugiere nuevos temas a tratar, reafirma o cuestiona la imagen del youtuber, etc. El youtuber sabe que no es nada sin sus seguidores, pues son ellos quienes hacen poderoso su canal por medio de sus likes y visualizaciones. Los youtubers insisten en que si la historia les ha gustado "le den al like" y son constantes sus muestras de agradecimiento por el número de visitas y suscriptores, una manera más de mantener y reforzar el vínculo creado con su audiencia.

Otras de las características fundamentales del estilo youtuber es el carácter artesanal o casero de los videos, grabados por lo general en el interior de una habitación con una cámara o una webcam. A pesar de que en los últimos años algunos youtubers han adoptado una estética más profesional y su éxito y popularidad ha hecho que cuenten con más medios y posibilidades para realizar producciones de alta calidad, siguen prefiriendo y conservando el formato casero de sus comienzos, de esta forma perpetúan la ideología del do it yourself o broadcast yourself, bajo la cual nació YouTube. En cualquier caso, la habitación del youtuber sigue siendo el principal escenario en el que se graba y desde el que se transmite el video, lo que contribuye a generar esa sensación de intimidad y familiaridad con la audiencia. De este modo, parece como si el youtuber dejara que su público penetre en su habitación, como si le "abriera la puerta" y le permitiera instalarse por unos minutos — los que dura cada video - en un espacio habitualmente íntimo, secreto y de acceso restringido, en especial para figuras como los padres. Al compartir este espacio íntimo, el youtuber consigue crear el ambiente propicio y reunir a la audiencia en torno a su relato, tal como el narrador conseguía reunir a la comunidad alrededor del fuego y hacer amenas las largas y frías noches de la antigüedad (Benjamin, 1991).

\section{¡Quiero ser youtuber!}

El éxito y la popularidad que han alcanzado en los últimos años muchos youtubers, cada vez más recogidos y amplificados por los medios de comunicación tradicionales, han hecho que muchos jóvenes alrededor del mundo aspiren actualmente a convertirse en youtubers. Ellos han sido testigos, en un contexto de crisis económica e incertidumbre laboral en muchos países, de cómo personas de su edad o unos años mayores se han hecho famosos publicando videos en YouTube, y lo más importante, de cómo han logrado transformar esta práctica o afición en una nueva profesión. Esto se refleja en encuestas recogidas por 
los medios de comunicación, como la de la consultora Adecco, según la cual ser youtuber se encuentra entre las diez profesiones más deseadas por los niños españoles. En el mismo sondeo emergieron con fuerza otras profesiones propias del mundo digital, como gamers, bloggers y community managers (Molina, 2016). Según otra encuesta de la revista Variety a adolescentes estadounidenses de entre 13 y 17 años de edad, en la que se preguntaba sobre los personajes más importantes del momento, ocho de los diez más votados fueron youtubers, por encima de celebridades del mundo del cine y la música, como Bruno Mars, Taylor Swift, Morgan Freeman, Meryl Streep, Will Smith o Johnny Deep (Ault, 2015). Estos datos, corroborados en muchas de las entrevistas realizadas en nuestro estudio, ponen de manifiesto la influencia que los youtubers ejercen en la actualidad en los adolescentes de distintas partes del globo, hasta el punto de haber generado en muchos de ellos el deseo y la ilusión de querer ser youtuber, ante la perplejidad que esto pueda causar en sus mayores.

Este deseo se sustenta en gran medida en la idea de que, si cualquier persona es capaz de subir videos a la plataforma, cualquiera puede, al menos de manera potencial, alcanzar el éxito y ganar dinero por ese medio. YouTube se creó bajo el ideal democrático - muy propio de la web 2.0 - de que todo el mundo puede subir videos a la plataforma y darse a conocer ahí. En este sentido, YouTube vino a democratizar el ecosistema mediático tradicional y nos permitió a "ti" y a todos "nosotros" —el you de YouTube, el you personaje de 2006 de la revista Time - distribuir "nuestros" videos en la plataforma de manera libre y gratuita, algo difícil de hacer en el anterior sistema vertical y jerarquizado de medios. De ahí el "utopismo radical" con el cual fue percibido YouTube desde sus comienzos, como puede observarse en la siguiente descripción de la plataforma, ofrecida por el diario The Independent en 2006:
YouTube es profundamente democrático. No hay ningún magnate de los medios determinando qué línea política deben tomar los clips. No hay bancos de ejecutivos inteligentes manipulando las emociones de sus presas. No hay directores artísticos de moda utilizando sus habilidades para vender productos. En lugar del mundo manipulado de Hollywood o de la televisión convencional, lo que ves es lo que eliges ver. Los videos que pones en el sistema son los videos que quieres poner en el sistema. No hay "persuasores ocultos" (McRae, 2006, citado en McMurria, 2006) [la traducción es nuestra].

Este artículo de The Independent, con el significativo título de "YouTube es joven, democrático y muestra que el mundo está cambiando ante nuestros ojos", señalaba una clara idealización de YouTube como una alternativa revolucionaria al dominio corporativo de los medios de comunicación tradicionales, una imagen que ha sido muy poderosa desde sus orígenes como plataforma (McMurria, 2006).

Este potencial democrático de ver y publicar los videos que uno quiera se extendió rápidamente al ámbito de la fama y la celebridad mediática y se activó el mito, presente hasta hoy, de que en YouTube todos podemos "difundirnos a nosotros mismos" — broadcast yourself — hacia la fama y la fortuna (Burgess y Green, 2009: 22), o en términos de Nick Couldry (2003), dar el salto de nuestro mundo cotidiano y "ordinario" —ordinary world — al deseado "mundo mediático" — media world-. Como matizan Burgess y Green (2009), esta promesa de traspasar el mundo ordinario y acceder al mundo de los medios y sus modos de representación está incrustada con firmeza en la propia plataforma, que incluso ha llevado a cabo competiciones e iniciativas de búsqueda de talento. En este punto, los autores se apoyan en la tesis del "giro demótico" de Turner (2010) para argumentar que más que una "democratización" de la cultura mediática, lo que observamos en casos como el de YouTube es más bien una especie de 


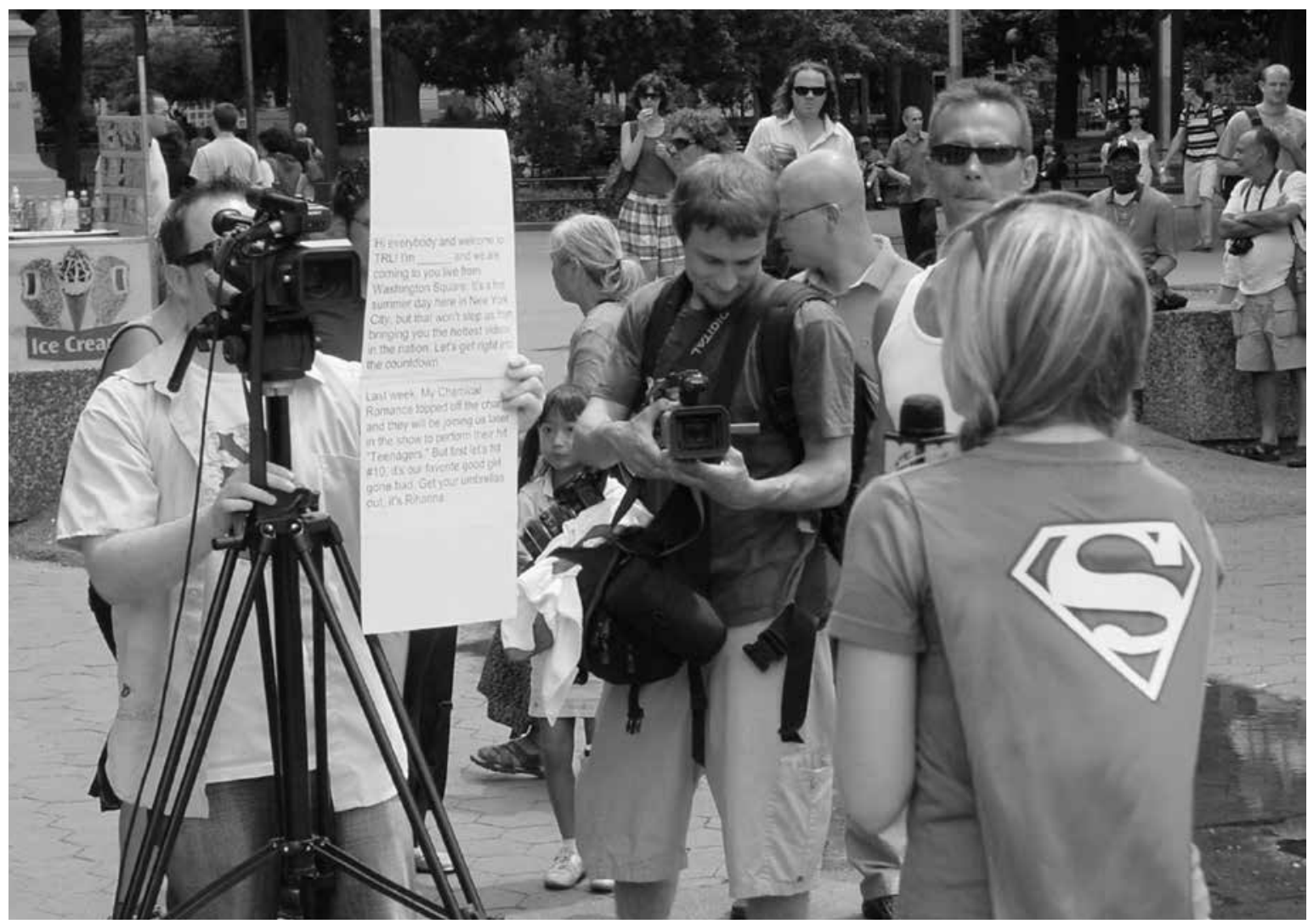

LARRY OSAN • Grabación para la serie Total Request Live de MTV en un evento de YouTube, julio de 2007.

“demotización”, es decir, una mayor visibilidad y participación de la gente común y corriente —ordinary people - en el mundo de los medios, pero no implica necesariamente una transferencia del poder mediático: "incluso cuando las personas comunes se convierten en celebridades por medio de sus propios esfuerzos creativos, no hay una transferencia necesaria de poder mediático: permanecen dentro del sistema de celebridad nativa de, y controlada por, los medios masivos" (Burgess y Green, 2009: 23). En este sentido, el "giro demótico" de la cultura mediática actual se apoyaría en las estructuras existentes de celebridad para ofrecer una especie de "celebridad ordinaria" que, "lejos de proporcionar alternativas a la industria de medios existentes, es producida y capturada por ella" (2009: 23).
La tesis del "giro demótico" es importante porque pone en entredicho la imagen de YouTube como espacio democrático de creación de fama y popularidad, eso a lo que muchos jóvenes —impulsados por la lógica del "yo podría ser ella o él”- aspiran hoy tras haber visto y seguido ejemplos como los de los youtubers mencionados y muchos otros. Esta tesis también hace hincapié en el mantenimiento de las estructuras de poder existentes y en la hegemonía económica y cultural que siguen detentando los medios tradicionales, a pesar de la supuesta revolución 2.0 y las redes sociales. Así, como señala Turner, por mucho que la gente común y corriente participe y se haga cada vez más visible en los medios, "las industrias mediáticas siguen controlando la economía simbólica” y "todavía se esfuerzan 
por operar esta economía al servicio de sus propios intereses" (2010: 16), algo que se observa claramente en el caso de los youtubers, como veremos a continuación.

\section{La apropiación capitalista del youtuber}

Al hablar de YouTube hay que distinguir entre el YouTube de antes y después de Google. ${ }^{1}$ Jin Kim (2012) ha señalado con claridad cómo a partir de que Google adquirió YouTube se produjo una "institucionalización" de la plataforma, que condujo a que este "nuevo" medio empezara a imitar o reproducir cada vez más la lógica y las reglas de los "viejos" medios. A partir de la compra de Google, más que competir, YouTube y los medios tradicionales representados por las grandes corporaciones empiezan a cooperar entre ellos de manera cada vez más activa. Un hecho muy significativo de esta cooperación fue la introducción en 2007 del YouTube Video Identification -Video ID-, un software desarrollado en colaboración con Disney y Time Warner que permite a los titulares de copyright —en su mayoría empresas de medios - encontrar en YouTube videos que infrinjan la autoría, bloquearlos y reclamar sus derechos. Al mismo tiempo, este software funciona como un eficaz dispositivo de vigilancia capaz de espiar a los usuarios del sitio y revelar sus datos y gustos personales a las corporaciones mediáticas.

Según Kim, esta cooperación entre YouTube y los medios tradicionales repercute en el propio contenido de la plataforma. Así, si el YouTube de antes de Google se caracterizaba por el auge de videos de aficionados en un entorno libre de anuncios, el YouTube de después de Google se distingue por la proliferación de videos profesionales, muchos de ellos procedentes de grandes compañías mediáticas, en un entorno que favorece la inserción de anuncios, que generan ingresos compartidos con estas compañías. El resultado de esta institucionalización de YouTube es la colonización corporativa de la plataforma, lo cual no es sino un ejemplo más de la colonización o apropiación capitalista de internet en su conjunto, y de la transformación, por lo tanto, de lo que parecía ser "una esfera pública cada vez más abierta, apartada del mundo del intercambio de mercancías", en "una esfera privada de mercados cada vez más cerrados, propietarios e incluso monopólicos" (McChesney, 2013: 96).

A partir de que Google compró YouTube, se ha ido convirtiendo en una especie de "corporatube”, como ha sugerido Toby Miller (2009: 427). Según este autor, lejos de representar el triunfo de un medio nuevo sobre otro viejo, lejos de ser una tecnología abierta y democrática, YouTube es más bien otro producto del capitalismo corporativo, un "valioso aliado de Hollywood" y de otros gigantes mediáticos. Miller cita incluso una frase significativa de Steve Chan, cofundador de la plataforma, en la que afirma que YouTube representa un "compromiso, no una interrupción” para el mundo corporativo, lo cual explicaría los miles de contratos que la plataforma ha firmado con los medios tradicionales a lo largo de estos años. Así, si hace tiempo la plataforma se caracterizaba por la proliferación de videos de aficionados, facturados con una estética artesanal y casera, últimamente "los videos más populares de YouTube provienen de corporaciones multimedia globales como Universal, Sony y Walt Disney" (Fuchs, 2014: 61). En este sentido, el propio Fuchs señala que medios sociales como YouTube no constituyen en realidad una esfera pública o un espacio democrático participativo, sino más bien espacios colonizados por grandes corporaciones, en especial por compañías multimedia que dominan, controlan y gestionan la atención y la visibilidad.

1 Jin Kim les Ilama "the pre-Google era" y "the post-Google purchase stage" (2012: 56). 
¿Cómo se relaciona todo esto con los youtubers? Nuestro argumento es que el fenómeno youtuber, como lo conocemos en la actualidad, se ha desarrollado principalmente en el YouTube de la era después de Google y constituye un ejemplo más de cómo las grandes corporaciones mediáticas están colonizando internet y aprovechando sus "viejas" estructuras de poder para apropiarse de "nuevos" medios como YouTube y de "nuevos" fenómenos de la cultura popular como el de los youtubers, al reproducir y perpetuar así su hegemonía económica y cultural.

El fenómeno youtuber empezó a tomar importancia cuando YouTube ya era parte activa del entramado de Google, el cual, como ha señalado Siva Vaidhyanathan, hace tiempo que ha dejado de ser un simple buscador de internet para convertirse en una "compañía general de medios de comunicación”, es más, en una de las instituciones globales más importantes del planeta, casi "indistinguible de la internet misma" (2012: 20). Para ser más exactos, el fenómeno de los youtubers cobró fuerza de verdad a partir de que YouTube decidiera ampliar su programa para Partners en 2012, ${ }^{2}$ el cual permitía a todos los creadores de videos la posibilidad de monetizar contenido en YouTube por medio de una amplia variedad de medios, pero de manera especial, con la inserción de anuncios en los propios videos - por medio de AdSense, plataforma de publicidad de Google- y la asociación con las denominadas networks o "redes multicanal", sobre las cuales nos detendremos en breve. En la propia página web de YouTube (Google, 2017a) se muestran algunas de las ventajas que el programa para Partners supuso para los creadores de contenido, por ejemplo, aumentar los propios ingresos y llegar a una audiencia global por medio de los videos monetizados, o la posibilidad de hacer un seguimiento del rendimiento y los ingresos de YouTube con YouTube Analytics. Este software de medición de audiencias habilitado por Google, permite al youtuber supervisar en todo momento el rendimiento de su canal y sus videos, le proporciona estadísticas e informes actualizados - como número de visitas de cada video, número de veces que ha sido compartido, datos demográficos de las visitas y edades, tipo de dispositivo desde el que acceden los usuarios, análisis de los comentarios obtenidos, etc. - con los que mide y analiza el éxito de su canal, y mantiene o rediseña su estrategia. YouTube Analytics puede entenderse como un dispositivo de vigilancia similar al YouTube Video Identification, pero al servicio del propio youtuber, quien puede conocer el público de su canal, su edad, sexo, ubicación geográfica y preferencia de contenidos, y orientar de manera estratégica sus temas a estos gustos y preferencias.

YouTube Analytics es una herramienta poderosa para cualquier youtuber, pues le facilita conocer a su audiencia hasta el máximo detalle y posicionar sus videos en la plataforma para que tengan éxito y sean rentables económicamente. Así, buena parte del éxito de los youtubers se debe también a la introducción de este dispositivo de vigilancia estratégicamente diseñado por Google, que en sí mismo funciona como un panóptico (Foucault, 1976), o más bien un "superpanóptico" (Poster, 1990) capaz de apropiarse de los datos de audiencia, los datos personales de estos youtubers, y en realidad, de todos aquellos que recurrimos a diario a su buscador y sus servicios asociados - YouTube, Gmail, Google Drive, Google Maps, Blogger, etc.- - Como señala Siva Vaidhyanathan:

Uno de los mayores atractivos de Google es que parece ofrecer gratis muchos servicios eficaces. Pero entre ella y sus usuarios existe una implícita

2

El programa para Partners de YouTube se creó en 2007, pero sólo podían beneficiarse de él los productores de contenido muy popular. En 2012, YouTube abrió el programa a todos los creadores de contenido, lo que amplió su uso y alcance, e hizo posible la emergencia de fenómenos como el de los youtubers. 
transacción no monetaria. Google nos proporciona las plataformas de búsqueda en la web, correo electrónico y Blogger, y videos en YouTube. A cambio, obtiene información sobre nuestros hábitos y predilecciones, para poder dirigirnos anuncios con mayor eficacia. Su actividad principal es hacer perfiles de consumidores. Genera expedientes de muchos de nosotros. Mete cookies en nuestro explorador de internet para rastrear nuestros clics y curiosidad [...]. Google es una caja negra. Sabe mucho de nosotros, y nosotros muy poco de ella (2015: 25).

Si el programa para Partners dio a los creadores de contenido la posibilidad de monetizar sus videos en la plataforma y hacer un análisis detallado y estratégico de su audiencia con YouTube Analytics - factores que impulsaron el fenómeno de los youtubers como lo conocemos hoy-, también introdujo la posibilidad de que los creadores de contenido se asociaran a las denominadas multi-channel networks (MCN) o redes multicanal. Las MCN son empresas que funcionan como intermediarios entre YouTube y los youtubers, y les ofrecen ayuda y asesoría en aspectos como gestión de contenido, promoción, obtención de ingresos, posicionamiento web, gestión de derechos de copyright, aumento de público, etc. A cambio, las MCN se quedan con un porcentaje de los beneficios del canal, que se distribuyen entre ésta, YouTube y el propio youtuber. Hoy, la mayoría de youtubers famosos forman parte de alguna de estas MCN, varias de las cuales han logrado acuerdos con importantes marcas y compañías comerciales. Las networks actúan como representantes de los youtubers, gestionan su carrera y negocian las condiciones de sus contratos con marcas. En los últimos años, sin embargo, muchas de estas MCN han sido adquiridas por grandes corporaciones mediáticas. El caso más representativo es el de Maker Studios, una de las networks más grandes e importantes del mundo - representante, entre otros, del famoso PewDiePie-, adquirida por Disney en 2014 por la espectacular cifra de 500 millones de dólares. El mismo año, el estudio de animación estadounidense DreamWorks Animation - adquirido en fechas recientes por NBC Universal por 3.8 millones de dólares- compró la network Big Frame por 15 millones de dólares. Dos años después, Warner Brothers adquirió la famosa network Machinima por 100 millones de dólares.

Estos ejemplos ponen de manifiesto que no sólo YouTube en su conjunto ha sido colonizado por las grandes corporaciones mediáticas (Miller, 2009; Fuchs, 2014), sino que fenómenos propios de esta plataforma, como los casos analizados de youtubers y networks que los representan, han sido objeto de apropiación de las de las mismas corporaciones capitalistas, lo que da lugar a una situación de control y hegemonía mediática no muy diferente a la de la era — predigital — de la comunicación masiva. Si bien la era digital ha generado nuevos modelos de negocio y ha propiciado el surgimiento de nuevos estilos comunicativos y profesiones, el caso de los youtubers pone de manifiesto el poder que siguen detentando las viejas corporaciones y estructuras mediáticas, que se adaptan a estos nuevos modelos y celebridades digitales y los adecuan a sus propios servicios e intereses (Turner, 2010). La convergencia mediática, en este sentido, no se produce sólo en una dirección, sino que las grandes compañías también se adaptan al nuevo ecosistema mediático (Kim, 2012), y en este caso, utilizan YouTube y a los youtubers como un nuevo recurso económico y una nueva herramienta de promoción, captación y expansión de audiencias. Desde esta perspectiva, queda lejos esa visión de YouTube como un espacio "revolucionario" y profundamente democrático en el que, según las palabras del periodista de The Independent citadas, no hay magnates, ejecutivos o directores de moda que utilizan sus habilidades para manipular y vender productos, un espacio en el que no hay, en definitiva, "persuasores ocultos". 


\section{Contrahegemonía youtuber}

Tras lo dicho hasta ahora, podemos observar con claridad cómo la corta historia de YouTube se caracteriza por un rápido y eficaz proceso de apropiación capitalista por parte de fuerzas más poderosas, como Google o Disney. Lo mismo ha sucedido en el caso específico de los youtubers, incluso de forma mucho más rápida. Esta transformación progresiva de YouTube en "corporatube" (Miller, 2009) —y de los youtubers en corporatubers, añadimos - parece estar generando una situación singular de concentración hegemónica de poderes económicos, políticos y culturales en la que encontramos, por un lado, un nuevo tipo de hegemonía digital propia del "capitalismo electrónico-informático" (Lins Ribeiro, en este volumen) representado por gigantes como Google, y por el otro, modelos hegemónicos heredados de la época de comunicación de masas anterior que consiguen adaptarse con eficacia al nuevo ecosistema mediático e imponer en él sus reglas y directrices.

Como advirtió Raymond Williams, "una hegemonía dada es siempre un proceso", no surge de modo pasivo como una forma de dominación, sino que "debe ser continuamente renovada, recreada, defendida y modificada". Asimismo, la hegemonía es "continuamente resistida, limitada, alterada, desafiada por presiones que de ningún modo le son propias". Por lo tanto, la realidad de toda hegemonía "es que, mientras que por definición siempre es dominante, jamás lo es de un modo total o exclusivo. En todas las épocas las formas alternativas o directamente opuestas de la política y la cultura existen en la sociedad como elementos significativos" (2000: 134). La hegemonía, si bien es siempre dominante, nunca se impone de manera absoluta, pues, como recuerda a su vez García Canclini:

En la circulación, y sobre todo en el consumo, los bienes y mensajes hegemónicos interactúan con los códigos perceptivos y los hábitos cotidianos de las clases subalternas. El repertorio de bienes y mensajes ofrecidos por la cultura hegemónica condiciona las opciones de las clases populares, pero éstas seleccionan y combinan los materiales recibidos - en la percepción, en la memoria y en el uso- y construyen con ellos, como el bricoleur, otros sistemas que nunca son el eco automático de la oferta hegemónica (1984: 75).

La cultura hacker y las comunidades de software libre son ejemplos paradigmáticos de fuerzas contrahegemónicas que cuestionan, responden y desafían la actual hegemonía tecnológica, política, económica y cultural. Un caso significativo en este sentido es el colectivo Anonymous, que se ha servido de servicios digitales como YouTube para dar a conocer sus actividades a favor de la libertad de expresión y la independencia de internet. De hecho, YouTube jugó un papel fundamental en la popularización de Anonymous como nuevo ente ciberactivista. La antropóloga Gabriela Coleman abre su etnografía sobre Anonymous, Las mil caras de Anonymous. Hackers, activistas, espías y bromistas (2016), con la descripción de un video colgado en YouTube el 29 de julio de 2007 por una entidad autodenominada Anonymous, desconocida en aquel momento para todo el mundo excepto para los cibernautas más expertos. Según Coleman, el video titulado "Querida Fox News" pretendía satirizar mediante el lulz —el tipo de humor macabro y descarriado de Anonymous - la caracterización hiperbólica que el canal de noticias había hecho de este colectivo en fecha reciente al presentarlo como "la máquina de odio de internet” y "los máximos proveedores de bromas pesadas y troleo en internet, 'hackers chutados de esteroides"' (2016: 9). Desde la publicación de este video en YouTube, Anonymous ha seguido difundiendo contenido en la plataforma para promover sus acciones - como el reciente video en el que le declaraba la "guerra total" a Donald Trump-, aunque muchos de estos videos han sido censurados y eliminados por la compañía propiedad de Google. 
El caso de Anonymous como fuerza contrahegemónica es interesante en otros aspectos, también señalados por Coleman. Según la autora, Anonymous representa un curioso y excelente ejemplo de "contramercantilización" en el que "si bien es verdad que Time Warner se lucra cada vez que alguien compra una máscara oficial de Guy Fawkes (Time Warner tiene el copyright de la película $V$ de Vendetta), Anonymous ha adoptado un símbolo popularizado por Hollywood y lo ha convertido en revolucionario" (Coleman, 2016: 236). ${ }^{3}$ Asimismo, Anonymous desafía el modelo hegemónico de celebridad individual hollywoodense - que sigue perpetuando la mayoría de youtubers - con la construcción de una celebridad colectiva que supone la eliminación de la persona y de todo lo asociado a ella en beneficio de un seudónimo colectivo protector, que actúa como una identidad común compartida. Como señala Coleman, en sus actividades de troleo y sus encarnaciones activistas, Anonymous actuaba como una fuerza colectiva incomparable con "la lógica impulsora de los medios de comunicación corporativos dominantes y las sensibilidades hegemónicas del yo" (2016: 51).

Volviendo al caso de YouTube y los youtubers, también es posible encontrar en la plataforma ejemplos de usuarios anónimos que portan la simbólica máscara de Guy Fawkes y hacen críticas en sus videos al fenómeno de los youtubers, las networks y las empresas que cada vez más gestionan su carrera y los representan. Un ejemplo es el canal "ME TOCA LOS COJONES - RESISTANCE TV”, en el que encontramos videos con títulos tan significativos como "YouTube, el control de masas perfecto", "Youtubers que piden dinero a sus suscriptores", "Boteando videos de YouTube”, "Audi España regala coches a youtubers infractores de tránsito", etc. Estos videos, publicados entre 2016 y 2017, son ejemplos de un tipo de discurso contrahegemónico que también es posible localizar dentro de la plataforma, aunque no de forma abundante, y que funciona como una especie de crítica desde dentro hacia todo el entramado digital, mediático y espectacular en el que se sustenta el fenómeno youtuber. En este tipo de videos podemos ver reflejados y explicados, a veces con demostraciones prácticas, aspectos no tan visibles ni "vendibles" de este fenómeno, por ejemplo, el empleo intencionado de bots - programas informáticos programados para imitar el comportamiento humano - para aumentar el número de visualizaciones, comentarios y suscriptores en un canal de YouTube, con el consiguiente aumento de ingresos y popularidad. Este tipo de contrahegemonía youtuber es importante porque revela los diversos mecanismos, estrategias y retóricas utilizadas por estos nuevos referentes juveniles a la hora de gestionar su fama, dinero y visibilidad, pues algunos han llegado a aprovechar su situación de privilegio para pedir dinero a sus suscriptores de manera explícita.

Junto a esta crítica contrahegemónica de oposición al sistema, cabe decir que existe en todo youtuber una posibilidad de rebelión y de resistencia. En este sentido, la figura misma del youtuber encarna la tensión entre hegemonía y contrahegemonía: plegarse a los deseos de las marcas y las grandes corporaciones mediáticas que los apoyan y financian, o mantenerse fieles a su extracción popular y seguir siendo "ellos mismos", esos chicos desenfadados y atrevidos que hicieron de su pasión un modo de vida, aspecto que constituye uno de los principales atractivos para sus jóvenes audiencias. El youtuber,

Guy Fawkes fue un conspirador británico del siglo xvII que participó en una conjura para volar la Cámara de los Lores en Londres y matar al rey protestante de Inglaterra. La máscara de Guy Fawkes se ha utilizado en un amplio número de protestas antigubernamentales y antisistema a raíz de que se popularizó con la serie de cómics $V$ de Vendetta, cuyo protagonista portaba una máscara similar, y sobre todo, a partir de su adaptación cinematográfica en 2006. La máscara de Guy Fawkes se ha convertido en el símbolo central del colectivo Anonymous, cuyos miembros siempre realizan sus acciones e intervenciones públicas tras ella. 


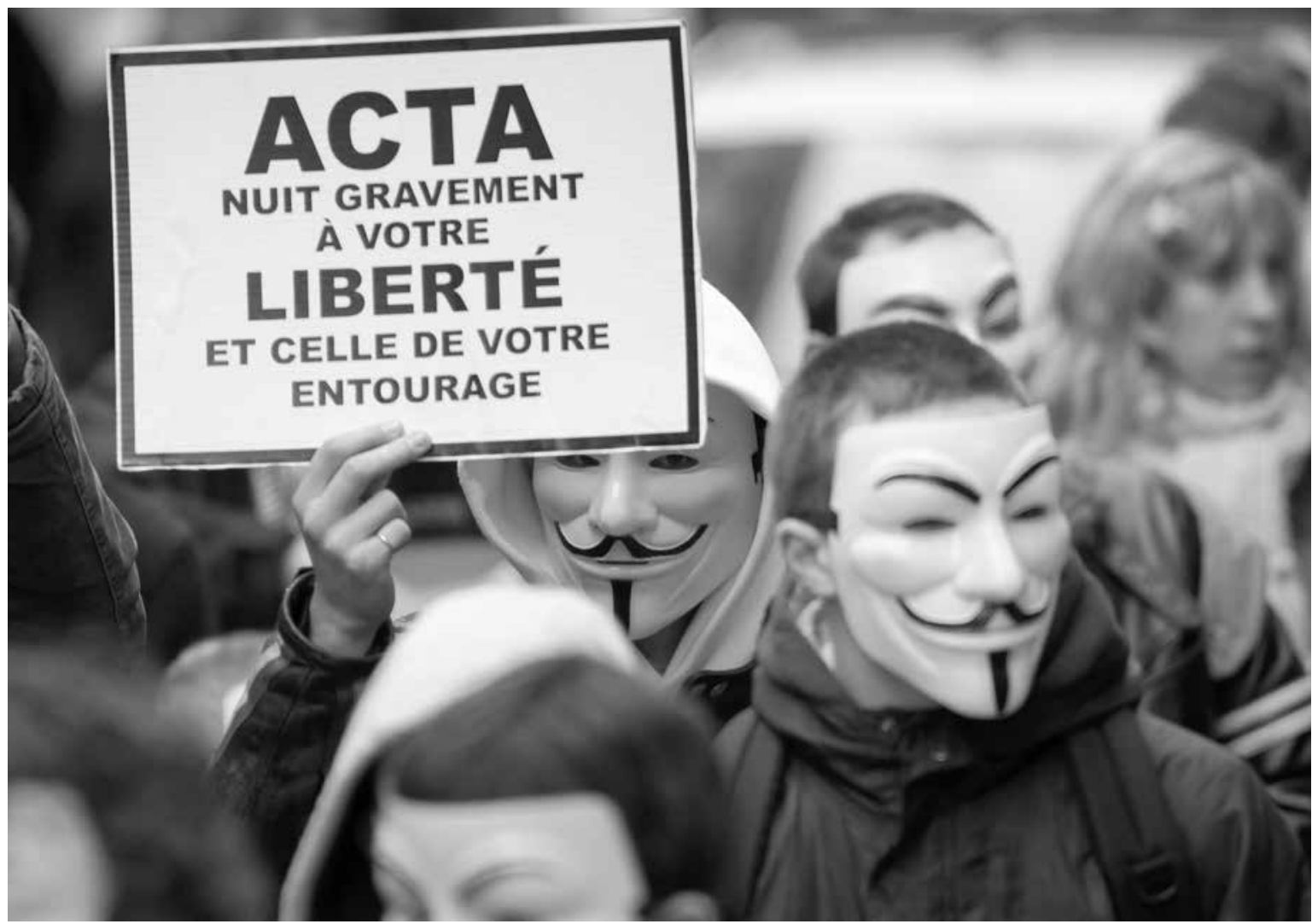

FRÉDÉRIC BISSON • Anonymous contra el Acuerdo Comercial Antifalsificación (ACTA, por sus siglas en inglés), en Rouen, Francia, marzo de 2012.

como decíamos al principio, construye y mantiene su audiencia por medio de las historias que cuenta en sus videos. En el caso de los youtubers españoles mencionados, se añade el empleo reiterado de un lenguaje ordinario que es parte fundamental de su estilo comunicativo - en términos de Mary Douglas (1998), de su "estilo de pensar"- En los casos de El Rubius, AuronPlay, Wismichu o Jordi Wild, el uso de palabras malsonantes es parte habitual de su repertorio, y según ellos, una forma directa y sin trabas de aproximarse a su público. En torno a este lenguaje, el youtuber construye un "estilo de pensamiento distintivo" que le permite ser original y mantener cotidianamente el nexo con su audiencia. Si el youtuber ve atacado su "estilo de pensar" particular, ése sobre el que ha ido construyendo su autenticidad como youtuber y fortaleciendo el vínculo con su audiencia, se rebela, critica y cuestiona las decisiones y mecanismos de la cultura hegemónica. En este sentido, un ejemplo característico se produjo en septiembre de 2016, cuando YouTube publicó nuevas normas de contenido "adecuado para anunciantes", que afectan el contenido mismo de los videos de los youtubers. Según esta nueva normativa, la plataforma de Google limitará los ingresos por publicidad a los autores de contenidos que considere "inadecuados", como el uso de un "lenguaje inapropiado, incluido el acoso, las obscenidades y el lenguaje vulgar", o las "escenas de carácter sugerente, incluidos desnudos parciales y humor verde" (Google, 2017b). Esta decisión generó un aluvión de críticas y protestas por parte de varios youtubers, 
quienes mostraron su completo desacuerdo ante el hecho de tener que "autocensurarse" y "cambiar su estilo" si quieren seguir subiendo videos a YouTube y generando ingresos con esta actividad. Una de las respuestas más comentadas fue la de El Rubius y su videoprotesta "La nueva censura en Youtube". En este video, con un uso ejemplar del humor y la ironía como forma de desacato, el popular youtuber realiza una aguda crítica a las nuevas normas de YouTube y concluye diciendo que seguirá subiendo los mismos videos de siempre, aunque le quiten dinero por su contenido inadecuado. Así lo explicó en la descripción del video:

He hecho este video porque había MUCHísimas personas preguntándome qué iba a pasar con mi canal después de las noticias sobre los cambios de normas en Youtube, y si iba a dejar de hacer videos o lo que sea. Don't worry. Tito Rubius subirá videos como siempre, y si hace falta que me "quiten" dinero para ser yo mismo, así será : $\mathrm{D}^{4}$

Este video y el elevado número de críticas y protestas que generaron las nuevas normas de contenido adecuado de YouTube ponen de manifiesto la importancia de ese "estilo de pensar" (Douglas, 1998) creado por los youtubers españoles y de las formas coloquiales y ordinarias - ahora consideradas inadecuadas por YouTube- de exponerlo, transmitirlo y compartirlo con su audiencia. Los youtubers perciben estas formas "inadecuadas" de expresarse como constitutivas de su identidad y autenticidad, de su "ser yo mismo", como dice El Rubius, formas que ellos defienden y reafirman en sus textos y videos mediante recursos como el humor y la ironía, tanto en el plano discursivo como en actuaciones o performances, y a pesar del rechazo y la censura de la cultura hegemónica que los cobija, controla y vigila. En definitiva, la cultura popular de estos y otros youtubers se ve coartada y manipulada por los valores hegemónicos de empresas y conglomerados mediáticos que dictan a los youtubers qué pueden decir y qué no, según su visión de lo que debe ser el entretenimiento familiar y el contenido "adecuado". En este sentido, como afirma Carlos Aguirre (2009: 128), las clases populares mantienen cierta iniciativa y poder de resistencia, pero siempre dentro de la interacción contradictoria con los grupos hegemónicos que controlan el mercado.

\section{Conclusiones}

A lo largo de este artículo hemos destacado varios aspectos relacionados con la figura emergente del youtuber, ese usuario de internet, por lo general joven, que ha alcanzado cierta fama y popularidad por los videos que sube de forma regular en YouTube. Si bien el fenómeno nació hace algunos años, en tiempos más recientes ha conseguido atrapar el interés de medios de comunicación de todo el mundo - en ocasiones con noticias cercanas al sensacionalismo - y ha pasado a formar parte del vocabulario y el imaginario popular de mucha gente. Nombres como PewDiePie, en los países anglosajones, o El Rubius, en los de habla hispana, están presentes hoy en las conversaciones y el imaginario de muchos niños y adolescentes que ven en ellos nuevos modelos a seguir, nuevas formas de adquirir fama y dinero por algo que "nosotros también podemos hacer": contar historias en formato video y publicarlas en internet o broadcast yourself.

Sin embargo, esta idea de que todos podemos publicar videos en YouTube $-\mathrm{y}$ de manera potencial, ser famosos y ganar dinero-, característica del tipo de retórica optimista y democrática de la web 2.0 y las redes sociales, choca con los cambios

$4 \quad$ Como parte del ejemplo del estilo de comunicación del youtuber, se ha respetado la grafía del texto original. Véase <https://www.youtube.com/watch?v=0xdv-fG|p9c>. 
que la plataforma ha experimentado al convertirse cada vez más en un espacio dominado por grandes corporaciones mediáticas que controlan y gestionan la atención y la visibilidad de ciertos contenidos. Lo mismo sucede en el caso de los youtubers. Los más populares han sido o están siendo contratados por las denominadas networks o redes multicanal. Al final, éstas han sido adquiridas por grandes corporaciones mediáticas. El caso más representativo es el de la famosa Marker Studios, que fue comprada en 2014 por Disney por la módica cifra de 500 millones de dólares.

Estos aspectos nos conducen a cuestionar la retórica democrática y participativa de la web 2.0 y las redes sociales, y a preguntarnos si la famosa "cultura de la convergencia" no se está inclinando cada vez más hacia el lado corporativo. Se trata de algo que Henry Jenkins había advertido en su exitoso Convergence Culture, en el que en un momento duda sobre "cómo mantener el potencial de cultura participativa tras la creciente concentración mediática", y "sobre si los cambios provocados por esta convergencia abren nuevas oportunidades de expresión o expanden el poder de los grandes medios" (2008: 22). Si bien en su libro Jenkins inclinaba claramente la balanza hacia el potencial participativo de los ciudadanos, y buena parte de la retórica optimista sobre la cultura participativa en el contexto académico se debe a este libro, el paso del tiempo parece inclinar la balanza hacia el otro lado, hacia un tipo de contratación mediática en el que gigantes como Disney se adaptan de manera rápida y eficaz al nuevo entorno digital y expanden su poder y hegemonía hacia plataformas como YouTube y fenómenos como el de los youtubers.

En otro lugar, respecto al caso específico de YouTube, Jenkins (2009: 124) reconoce su deuda con McMurria (2006) y señala cómo la cultura participativa no es necesariamente una cultura diversa, y que si bien los mecanismos de YouTube parecen democráticos, en realidad ocultan contenidos y perspectivas de las minorías en beneficio de la cultura mainstream, los medios de comunicación y las corporaciones que insistentemente la producen y sustentan. Es cierto que estos contenidos circulan en YouTube, pero son empujados hacia los márgenes, mientras otros contenidos son impulsados hacia posiciones más visibles y dominantes. Lo mismo sucede con el tipo de discursos contrahegemónicos que critican el propio funcionamiento de YouTube, Google y los youtubers. Este contenido crítico circula por la plataforma, pero es más difícil de localizar que el de youtubers apoyados por grandes empresas y corporaciones, que son cada vez más numerosos. En este sentido, YouTube permite la existencia y creación de discursos contrahegemónicos en su seno. Pero también ejerce un control sobre estos por la posibilidad de censurarlos o eliminarlos en cualquier momento. De hecho, puede limitar o censurar cualquier tipo de contenido ya sea con el argumento de velar por la integridad moral de la ciudadanía — como vimos respecto a las nuevas normas de contenido adecuado-, por sus propios intereses o por los de las corporaciones con las que trabaja y coopera de forma efectiva —Disney, Time Warner, Sony, Universal, etc.- - Así, esta nueva hidra hegemónica permite expresarnos y hacer circular nuestros videos y producciones por medio de sus redes, pero en un estado de vigilancia y control permanentes, que busca mitigar el desafío contrahegemónico omnipresente. Tal es, según Williams (2000), la condición misma de todo proceso hegemónico, continuamente renovado y continuamente resistido. D 


\section{Bibliografía}

Aguirre, Carlos, 2009, "Hegemonía”, en Mónica Szurmuk y Robert Mckee Irwin (coords.), Diccionario de estudios culturales latinoamericanos, Siglo XXI Editores, México, pp. 122-128.

Ault, Susanne, 2015, "Digital Star Popularity Grows versus Mainstream Celebrities”, en Variety, 23 de julio. Disponible en línea: <http://variety. com/2015/digital/news/youtubers-teen-survey-ksi-pewdiepie-1201544882/>.

Benjamin, Walter, 1991, "El narrador", en Walter Benjamin, Iluminaciones IV. Para una crítica de la violencia y otros ensayos, Taurus, Madrid, pp. 111-134.

Burgess, Jean y Joshua Green, 2009, YouTube. Online Video and Participatory Culture, Polity Press, Cambridge.

Coleman, Gabriela, 2016, Las mil caras de Anonymous. Hackers, activistas, espías y bromistas, Arpa, Barcelona.

Couldry, Nick, 2003, Media Rituals: A Critical Approach, Routledge, Londres y Nueva York.

Douglas, Mary, 1998, Estilos de pensar. Ensayos críticos sobre el buen gusto, Gedisa, Barcelona.

Foucault, Michel, 1976, Vigilar y castigar. Nacimiento de la prisión, Siglo XXI Editores, México.

Fuchs, Christian, 2014, Social Media. A Critical Introduction, Sage, Londres.

García Canclini, Néstor, 1984, "Gramsci con Bourdieu. Hegemonía, consumo y nuevas formas de organización popular”, en Nueva Sociedad, núm. 71, pp. 69-78.

Google, 2017a, “Descripción general del Programa para Partners de YouTube”. Disponible en línea: <https://support.google.com/youtube/ answer/72851?hl=es>.

_ 2017b, "Directrices de contenido adecuado para anunciantes". Disponible en línea: <https://support.google.com/youtube/ answer/6162278?hl=es>.

Jenkins, Henry, 2008, Convergence Culture. La cultura de la convergencia de los medios de comunicación, Paidós, Barcelona.

2009, "What Happened before YouTube", en Jean Burgess y Joshua Green, YouTube. Online Video and Participatory Culture, Polity Press, Cambridge, pp. 109-125.

Kim, Jin, 2012, "The Institutionalization of YouTube: From User-Generated Content to Professionally Generated Content”, en Media, Culture \& Society, vol. 34, núm. 1, pp. 53-67.

Lafkioui, Mena, 2008, "Reconstructing Orality on Amazigh Websites”, en Mena Lafkioui y Daniela Merolla (eds.), Oralité et nouvelles dimensions de l'oralité. Intersections théoriques et comparaisons des matériaux dans les études africaines, Publications Langues'O, pp. 111125. Disponible en línea: <https://hal.archives-ouvertes.fr/hal-01111419/document>.

Leith, Sam, 2012, ¿Me hablas a mí? La retórica desde Aristóteles hasta Obama, Taurus, Barcelona.

Martín-Barbero, Jesús, 1987, De los medios a las mediaciones: comunicación, cultura y hegemonía, Gustavo Gili, Barcelona.

McChesney, Robert W., 2013, Digital Disconnect. How Capitalism is Turning the Internet against Democracy, New Press, Nueva York.

McMurria, John, 2006, "The YouTube Community”, en Flow, 20 de octubre. Disponible en línea: <https://www.flowjournal.org/2006/10/theyoutube-community-2/>

McRae, Hamish, 2006, "YouTube Is Young, Democratic and Shows that the World Is Changing before Our Eyes", en The Independent, 10 de octubre. Disponible en línea: <http://www.independent.co.uk/voices/commentators/hamish-mcrae/hamish-mcrae-youtube-isyoung-democratic-and-shows-that-the-world-is-changing-before-our-eyes-419510.html>.

Miller, Toby, 2009, “Cybertarians of the World Unite: You Have Nothing to Lose but Your Tubes!”, en Pelle Snickars y Patrick Vonderau (eds.), The YouTube Reader, National Library of Sweden, Estocolmo, pp. 424-440.

Molina, Javier, 2016, "Los más pequeños quieren ser de mayor 'youtubers' y 'gamers'”, en El Mundo, 27 de julio. Disponible en línea: <http:// www.elmundo.es/economia/2016/07/26/5797453722601dc02a8b462f.html>.

Poster, Mark, 1990, The Mode of Information, Polity Press, Cambridge.

Turner, Graeme, 2010, Ordinary People and the Media: The Demotic Turn, Sage, Londres.

Vaidhyanathan, Siva, 2012, La googlización de todo (y por qué deberíamos preocuparnos), Océano, México.

Williams, Raymond, 2000, Marxismo y literatura, Península, Barcelona. 